\title{
Capitalismo global y petroficción en Temporada de huracanes de Fernanda Melchor
}

\section{Global Capitalism and Petrofiction in Fernanda Melchor's Hurricane Season}

\author{
Francesco Di Bernardo \\ Benemérita Universidad Autónoma de Puebla, México \\ francesco.dibernardo@outlook.com
}

Resumen: El presente artículo discute la novela de Fernanda Melchor Temporada de huracanes (2017) analizando, a través de las teorías de Silvia Federici y Rita Laura Segato, el valor simbólico de algunos personajes catalizadores de la crítica social del modelo socioeconómico extractivo y neoliberal. Asimismo, el artículo evidencia el papel de la extracción de los recursos energéticos en la disgregación del tejido social que la novela describe y discute las analogías entre la novela de Melchor y la petroficción. La discusión está enmarcada en el contexto de recientes contribuciones sobre narconarrativas, subrayando las diferencias entre estas y las descripciones de la violencia en la novela de Fernanda Melchor.

Palabras claves: Melchor, literatura mexicana, petroficción, realismo, Veracruz.

Abstract: The present article discusses Fernanda Melchor's novel Hurricane Season (2017) analyzing through the theories of Silvia Federici and Rita Laura Segato the symbolic value of some characters which work as catalysts for social criticism of the extractive and neoliberal socio-eco- 
nomic model. Likewise, the article shows the role of the extraction of energy resources in causing the disaggregation of the social fabric that the novel describes and discusses the analogies between Melchor's novel and the "petrofiction". The discussion is framed in the context of recent contributions on narconarratives, highlighting the differences between these and the descriptions of violence in Fernanda Melchor's novel.

Keywords: Melchor, Mexican Literature, Petrofiction, Realism, Veracruz.

Recibido: Io de mayo de 2020

Aceptado: 5 de enero de 2021 https://dx.doi.org/I O.I 5 I 74/rv.vi4i29.548

T a narrativa de Fernanda Melchor, nacida en 1982 en Veracruz, Lestá inevitablemente vinculada a la historia reciente del estado del Golfo de México. Su escritura expresa un compromiso con el contar la sociedad veracruzana en sus diferentes matices, incluso los más sórdidos y oscuros: pobreza, negligencia social y la inseguridad. Fernanda Melchor inicia a escribir como periodista, actividad por la cual recibe varios premios, tales como el Primer Certamen de Ensayo sobre Linchamiento, organizado por la Comisión Nacional de Derechos Humanos (CNDH) en 2002, y el Premio Estatal de Periodismo, en 2009. El libro de crónicas Aqui no es Miami (2013) es quizás el ápice de su escritura de no ficción. En 2008, publica una obra de literatura infantil, Mi Veracruz, mientras que su primera novela Falsa liebre se publica en 2013. Esta novela se centra en personajes que pueblan los márgenes de una sociedad abandonada por las instituciones políticas y económicas. Alejandro Badillo alaba la novela por una prosa que evoca a Cormac McCarthy y escribe que la novela es "una contestación que rescata el poder de la ficción y su capacidad para retratar el mundo actual" (2014). Sin embargo, 
Falsa liebre no obtiene el éxito de la siguiente y más reciente novela, Temporada de huracanes (2017), que ha sido traducida en varios idiomas y ampliamente elogiada por la crítica. Al respeto, Antonio Ortuño escribe de la novela: "[p] or fin gran narrativa, ambiciosa, rotunda, con todos los matices y todas las letras", mientras que Edmundo Paz Soldán llama la atención sobre la forma narrativa, que evoca El otoño del patriarca (1975) de Gabriel García Márquez por el uso de párrafos largos y casi ininterrumpidos a lo largo de la novela. El escritor boliviano agrega: "Melchor despliega una prosa que convierte la oralidad en poesía, en la que las malas palabras, el deseo de nombrar lo obsceno y lo escatológico, se revelan en toda su explosiva belleza" (2017).

Temporada de huracanes comienza con la crónica del hallazgo de un cadáver en un río. El cadáver hinchado y en descomposición de la Bruja, el personaje alrededor del cual se construye la trama de la novela, emerge del agua amarillenta y espumosa de un canal y es avistado por un grupo de cinco adolescentes que se habían aventurado por el barroso curso de agua atraídos por la presencia de lo que desde lejos parecía un objeto misterioso e inidentificable (Melchor, 2017: 1-2). En una primera lectura, la novela "[parece] responder a un catálogo obvio, una especie de programa para la novela [mexicana] actual [...] el machismo salvaje, la narcocultura [...], el fracaso educativo, el alcoholismo, y desde luego el trópico, los huracanes y el carnaval” (Wolfson, 2017). Efectivamente, la novela se caracteriza por la presencia de policías corruptos y otros arquetipos del género de la narconovela, como las pandillas que causan estragos en el tejido social y los políticos coludidos con los cárteles. Sin embargo, la narcoiconografía, una representación de un mundo "dominado por los actos inhumanos de los malhechores [...] que luchan entre sí hasta la muerte" (Zavala, 2014: 345), es solo una característica marginal de la novela. La principal preocupación de la novela es, en cambio, las condiciones 
sociales que se producen en el área geográfica en la que los eventos tienen lugar y en donde se mueven los protagonistas. Este enfoque aleja la novela de los tropos de narconarrativas, que explican la narcoviolencia como resultado de la corrupción generalizada de funcionarios públicos y políticos. Estas narrativas, tanto en el ámbito literario como periodístico, limitan la comprensión del fenómeno de las emergencias de la violencia relacionada con las drogas, "merma[n] nuestra comprensión de las transformaciones históricas de los discursos oficiales de la violencia y [...] despolitiza[n] las dificultades más urgentes sobre desigualdad social" (Zavala, 2016: 194). Temporada de huracanes subvierte el topos de la narconovela, ya que no se basa en dicotomías sociedad civil contra estado corrupto y cárteles. Además, mientras que la narconovela se centra en el estilo de vida y los hechos de los sicarios y los jefes de los grupos criminales, así como en la "ecología de las relaciones entre las personas que habían sostenido, durante décadas, las esferas más bajas [...] de los ciclos comerciales informales" (Herlinghaus, 2013: 59), la novela de Melchor arroja una luz sobre lo que le sucede a las víctimas de lo que Segato llama "segundo estado" (la combinación de poderes corporativos, estatales y criminales) (2010: 70). De hecho, las historias de las víctimas, personajes como Norma, Bruja, e incluso Luismi, siempre están en el centro de la narración de la novela, mientras los que podrían considerarse personajes arquetípicos de la narconovela quedan relegados a los márgenes narrativos. Al desviar la atención de los narcotraficantes, los políticos corruptos y los agentes de policía y llamando la atención sobre los espacios de precariedad en el que prolifera la violencia, Temporada de huracanes politiza el discurso alrededor de la violencia. Al centrarse en las víctimas y en los efectos sociales de la negligencia, las desigualdades y la explotación, la novela de Melchor adquiere un poder de subversión del discurso narconarrativo aún más evidente ya que normalmente las víctimas tienden a permanecer sin 
nombre, no reconocidas y relegadas en una bruma de olvido -una realidad, sin embargo, evocada en la novela, ya que "nadie en el pueblo supo nunca cómo se llamaba aquella pobre endemoniada [Bruja]" (Melchor, 2017: 33)- mientras que los perpetradores tienen prominencia en los medios, en la cultura popular, así como en la cinematografía y la literatura representaciones dominadas por lo narcoimaginario. En otras palabras, Temporada de huracanes:

[pulveriza] la posibilidad de las grandes explicaciones unívocas, aquellas que, desde el gobierno o los partidos políticos hasta la mayoría de las columnas periodísticas, suelen malabarear un concepto o un término mágicos con que dar cuenta cabal de la descomposición social del país, sin conocer la historia particular de las regiones, los factores añejos y los apenas incorporados, los usos particulares (Wolfson, 2017).

La protagonista de la novela es, por lo tanto, precisamente la "historia particular" de La Matosa, una ciudad ficticia quizás identificable con los distritos petroquímicos del estado de Veracruz. La dinámica socioeconómica específica y las relaciones de poder producidas por las fuerzas del capitalismo global, así como la violencia inherente de la actividad extractiva y su logística en Veracruz son el foco de Temporada de huracanes. En este estado, la masiva presencia la industria extractiva produce una configuración de las relaciones de poder arraigadas en la transformación del territorio y de la vida de sus habitantes. Tierras tropicales, anteriormente empleadas para actividades agrícolas, han sufrido deforestación y han sido reemplazadas por una infraestructura imponente caracterizada por la presencia de quemadores que liberan gas a la atmósfera, conductos de petróleo que derraman contaminantes en el sistema de agua y una compleja disposición de válvulas y tuberías que generan contaminación acústica. La transición de la agricultura tropical a 
la industria extractiva también ha significado la transformación de los campesinos en mano de obra asalariada y la transferencia forzada de población (Checa-Artasu, 2014: 7-10). Además, en 2013, el Congreso aprobó cambios a la constitución para permitir la apertura de la industria de hidrocarburos del país a empresas transnacionales privadas con la intención de atraer inversiones extranjeras para aprovechar los recursos energéticos del país (Paley, 2014: 8). La parte sur del Estado, debido a su relevancia geoestratégica (ubicada en el istmo de Tehuantepec que conecta el Atlántico con el Pacífico) fue declarada Zona Económica Especial (ZEE). Estos últimos brindan espacios de libertad de movimiento para el capital transnacional: a las corporaciones se les otorgan facilidades fiscales, aduaneras, logísticas y garantías para acceder a activos estratégicos comunales y a la fuerza laboral local, mal pagada (Avila y Avila Romero, 2018: 149-151). Las premisas de la imposición de ZEE se basan en lógicas coloniales según las cuales los espacios indígenas y campesinos son calificados como subdesarrollados, espacios vacíos en juego. En este sentido, dado que la realidad social de Veracruz se ve tan inevitablemente afectada por la industria de extracción, la perspectiva de la novela sobre la realidad social está inevitablemente informada por el papel de ese modelo económico en la reconfiguración del tejido social de Veracruz. Temporada de huracanes, por ende, recupera características temáticas de la petrofiction, concepto acuñado por Amitav Ghosh en su ensayo-reseña Petrofiction: The Oil Encounter and the Novel de 1992 de la novela de Abdelrahman Munif, Cities of Salt (1984), que trata de "la transición de una formación social tradicional a una forma de vida colonial-capitalista" (Riddle, 2018: 57; la traducción es mía). El petróleo, en cuanto "producto básico, una sustancia de la que depende el mundo", ha tenido un impacto tan enorme en la vida humana, que es "una parte consciente de nuestros imaginarios sociales" (Szeman, 2012: 3; la traducción es mía). Como parte del imaginario social, aunque 
la novela no mencione directamente la estructura socioeconómica e industrial, los efectos del modelo socioeconómico dominante en Veracruz y de la industria extractiva forman de lo que Jameson denomina el "inconsciente político" de la novela. Según la teoría de Jameson, la literatura, en cuanto acto simbólico social, es influenciada por la totalidad histórica y los síntomas de una precisa condición sociohistórica se revelan a nivel del inconsciente (1981: 1-88). Asimismo, la ausencia de los recursos naturales como mercancía en la ficción literaria en general es una práctica que refleja la eliminación de la materialidad de la extracción del discurso público. Bajo las lógicas del capitalismo tardío, la extracción de recursos y las relaciones sociales que produce en los espacios donde se despliega se perciben como acciones ineludibles de elementos naturales, mientras que la actividad extractiva del modelo económico parece "estar impulsada por fuerzas internas invisibles en su autosostenibilidad" (Riddle, 2018: 57-58; la traducción es mía). En la novela, la crítica de la "naturalización" de las prácticas extractivas del capitalismo hace uso de la evocación al poder destructivo de los huracanes como metáfora del impacto de la explotación de los recursos naturales en las sociedades latinoamericanas y su ambiente en Cien años de soledad (1967), cuando se cuenta que Macondo será arrasado por "un pavoroso remolino de polvo y escombros centrifugado por la cólera del huracán bíblico” (843). En la novela de Melchor, símilmente, se cuenta que:

[...] las malas vibras son las culpables de tanta desgracia: decapitados, descuartizados, encobijados, embolsados que aparecen en los recodos de los caminos o en fosas cavadas con prisa en los terrenos que rodean las comunidades. Muertos por balacera y choques de autos y venganzas entre clanes de rancheros; violaciones, suicidios, crímenes pasionales como dicen los periodistas (Melchor, 2017: 217). 
La evocación del realismo mágico garciamarqueziano y el uso de la simbología de la fuerza destructiva de los vientos y de una naturaleza impregnada por amenazas aparentemente sobrenaturales sirve para delinear el espacio de la novela. Asimismo, la interpretación sobrenatural de la fuerza de los huracanes evoca la "trilogía bananera" de Miguel Asturias, en la cual "the use of an irrealist, liminal event", como el huracán sirve a la comunidad local para "rearrange $[\ldots]$ the conditions of the possible" (Deckard, 2016: 37), tratar de explicarse los trastornos sociales que el capitalismo global desata en su territorio. La Matosa, por ende, es descrito como lo que Yi Fu Tuan llama en su homónimo libro "paisaje de miedo", un lugar donde se expresan "las infinitas manifestaciones de las fuerzas del caos" (2013, 6; la traducción es mía). La Matosa, como Macondo, representa un espacio mítico de la depredación de las fuerzas extractivas del capitalismo:

La Matosa, represents the type of peripheral community that would have played a secondary role in Mexico's oil-fueled development projects. It grows by creating a buoyant but precarious parallel market of brothels and bars that quickly falls victim to neoliberal austerity policies, economic crisis, and the rise of the drug trade (Sánchez Prado, 2020).

En el caso de la novela de Melchor, tal como en Cien años de soledad, en el cual el pavoroso remolino representaba el pillaje de América Latina por prácticas neoimperialistas del capitalismo, la fuerza del caos son precisamente las prácticas extractivas del capitalismo global. Temporada de huracanes, como petronovela, saca a la superficie (en el espacio de La Matosa) y evidencia las consecuencias de un modelo socioeconómico de acumulación por desposesión (Harvey, 2004: 64) impuesto a las comunidades del estado mexicano de Veracruz. La Matosa/Veracruz es una "comu- 
nidad desimaginada", una comunidad sacrificada por fines de desarrollo capitalistas y por el modelo de extracción (Nixon, 2013: 150; la traducción es mía). La novela proporciona una crítica de las formas de violencia que Rob Nixon llama slow-violence (2013: 2), una forma de violencia que no se manifiesta solo de manera evidente, sino que se desenvuelve con el tiempo a través de una lenta pero inexorable depredación socio-económica y ambiental, a través de mecanismos tales como privatizaciones, reducciones de derechos laborales, explotación laboral y ambiental, contaminación, recortes de presupuesto para servicios sociales, etc., que traen como consecuencia la fragmentación del tejido social. Esta forma de violencia, que se manifiesta a través de la falta de intervención social, afecta la comunidad depredada que es, por último, "physically unsettled and imaginatively removed" (Nixon, 2013: 150151) o como se alude en la novela, arrasada por un huracán.

En la novela, los efectos del modelo extractivo y neoliberal, como totalidad sociopolítica subyacente, son reflejados en las descripciones de la disgregación social y a través de la historia de sus personajes, como Bruja, Norma y Luismi, personajes fundamentales para la crítica que la novela propone del dominio territorializado del capital transnacional y sus prácticas de cercamiento y privatización de las tierras comunales por fines de extracción.

Bruja, una mujer pobre, marginada, transgénero, personifica la condición de víctima de los procesos de desintegración social; una figura que testifica cómo la combinación del proceso de desmantelamiento de "regímenes comunitarios" va de mano con "la demonización de miembros de las comunidades afectadas" (Federici, 2018: 15). Sin embargo, la figura de la bruja no remite exclusivamente a una idea de victimización, ya que las brujas representan "la resistencia que las mujeres opusieron a la difusión de las relaciones capitalistas" (Federici, 2004: 233) a través del poder que habían obtenido en virtud de su sexualidad, su control sobre 
la reproducción y su capacidad de curar. La Bruja de Temporada de huracanes -que siendo una mujer transgénero desafía también las normas patriarcales y el machismo resultante de la militarización del lugar- ofrece una resistencia a la fragmentación del tejido social determinado por la imposición del individualismo salvaje del capitalismo ya que, por ejemplo, suele ofrecer soporte, comida y techo a las mujeres explotadas en prácticas de prostitución.

El cuerpo de la Bruja, que emerge de las aguas sucias del "canal de riego del Ingenio" (Melchor, 2017: 32), denuncia la condición de abandono que afecta a los espacios de precariedad como La Matosa/Veracruz evocando de manera paralela la emersión del petróleo del subsuelo. Del mismo modo, la referencia a un río contaminado y "oleoso" recuerda los derrames de petróleo que han afectado los cursos de agua, una manifestación de la violencia lenta que afecta a sujetos tales como la Bruja, considerados desechables y expulsados de sus territorios a través de mecanismo de "cercamiento" moderno, forma de territorialización del capital. La palabra "cercamiento" refiriere a las primeras formas de privatización de las tierras comunales en la Europa del siglo xv, que causaron la expulsión de campesinos pobres y la pérdida de sus formas de sustento:

En Europa, a fines del siglo xv, coincidiendo con la expansión colonial, comenzó la privatización de la tierra que se implementó de distintas formas: expulsión de inquilinos, aumento de las rentas e incremento de los impuestos por parte del Estado, lo que produjo el endeudamiento y la venta de tierras (Federici, 2004: 99).

Según Federici, los arquetipos negativos asociados a las figuras de las brujas/curanderas, que servirán también para justificar la caza de brujas, acompañan el fenómeno de expropiación de las tierras. La caza de brujas fue un fenómeno predominantemente rural y, como tendencia, afectó regiones en las que la tierra había 
estado o estaba siendo cercada. Los fenómenos de demonización de la bruja y de la caza, sin embargo, no fueron limitados a Europa, sino que:

[1] a caza de brujas y las acusaciones de adoración al Demonio fueron llevadas a América para quebrar la resistencia de las poblaciones locales [...]. El destino común de las brujas europeas y de los súbditos coloniales está mejor demostrado por el creciente intercambio, a lo largo del siglo XviII, entre la ideología de la brujería y la ideología racista que se desarrolló sobre el suelo de la Conquista (Federici, 2004: 273).

También en las colonias americanas, las mujeres eran más vulnerables a ser acusadas de brujería. Se podría pensar, por ejemplo, en la leyenda nacida en tierra veracruzana, la Mulata de Córdo$b a$, que relata las formas de racismo a las cuales fueron sujetas las mujeres afrodescendientes durante la colonia y que se expresaron en procedimientos judiciales por acusaciones de brujería (Prado, 2013). En el contexto del mundo globalizado de hoy, Federici explica que, en América Latina, como en algunos países africanos, el renovado "surgimiento" de brujas:

ha acompañado la pérdida de posición social de las mujeres provocada por la expansión del capitalismo y la intensificación de la lucha por los recursos que, en los últimos ańos, se ha venido agravando por la imposición de la agenda neoliberal [...] Si aplicamos, sin embargo, las lecciones del pasado al presente, nos damos cuenta de que la reaparición de la caza de brujas en tantas partes del mundo durante las décadas de 1980 y 1990 constituye un síntoma claro de un nuevo proceso de "acumulación primitiva", lo que significa que la privatización de la tierra y de otros recursos comunales, el masivo empobrecimiento, el saqueo y el fomento 
de la divisiones de comunidades que antes estaban cohesionadas han vuelto a formar parte de la agenda mundial (2004: 312-314).

Las brujas, en realidad, son mujeres pobres, a menudo de edad mayor, que han sido expulsadas de sus tierras y que, en un contexto de rígida división de roles de género, tienen que recurrir a algunas formas de trabajo informal para sobrevivir, ya que la fragmentación de sus comunidades las ha dejado sin soporte (Federici, 2018: 16-18). Debido a la ruptura de su rol de género, estas mujeres se vuelven socialmente aisladas y víctimas de prejuicios por parte de los demás habitantes (Federici, 2018: 24-33). En Temporada de huracanes se encuentran referencias al asunto de estos prejuicios. Por ejemplo, Bruja es objeto de rumores por parte de algunos de los pobladores, particularmente hombres, de La Matosa, que insinúan que en su casa se organizan rituales orgiásticos y se practican maleficios. Inclusive su identidad y nacimiento es objeto constante de voces incontroladas, como las que aseguran que en realidad ella no fue parida por su madre (Bruja vieja) sino que esta la robó de otras personas. Uno de los comentarios que se escucha con más frecuencia en el Sorajuana, bar en el cual se reúnen los hombres del pueblo es que: "la Vieja nomás estaba loca y (que) a la Chica seguramente se la había robado de alguna ranchería" (Melchor, 2017: 21). Asimismo, se insinúa que Bruja vieja había matado a su esposo para robarle el tesoro que subsecuentemente habría escondido en la casa. Sin embargo, ella "juraba que no era cierto, que no había ningún tesoro, que ella vivía de la renta de las tierras que quedaban, unas parcelas repartidas alrededor de la casa en donde el sindicato del ingenio sembraba cańa” (Melchor, 2017: 32), terrenos que probablemente son objeto de interés de corporaciones ya que "witchcraft accusations are more frequent in areas designated for commercial projects or where land privatization processes are underway [...] and when the accused have some land that can be 
confiscated" (Federici, 2018: 50). Los crímenes que se atribuyen a las brujas son el resultado de la pobreza o simplemente estereotipos con los cuales se suele criminalizar a las mujeres pobres como Bruja vieja para expulsarlas de sus tierras. Ella, en realidad, a pesar de los rumores sobre su supuesto tesoro, "vivía [...] en un cuchitril [...] pura pinche basura" (Melchor, 2017: 32). Al igual que la historia del tesoro, las acusaciones de brujería en contra de individuos vulnerables y aislados socialmente como las Brujas se explican con la búsqueda de un chivo expiatorio responsable de utilizar poderes ocultos para acumular la riqueza que la comunidad ha visto desaparecer del territorio (Federici, 2004: 317), recursos que en realidad han sido trasladados hacia los centros de poderes económicos transnacionales. En este contexto socioeconómico, "brutalizing women is functional to the 'new enclosures'. It paves the way for the land grabs, privatizations, and wars that for years have been devastating entire regions" (Federici, 2018: 50). En efecto, contrariamente a lo que se creía en el pueblo, la Bruja no había sido robada de otra familia, sino que su madre se había quedado embarazada después de haber sido víctima de violencia sexual por parte de un grupo de criminales llegado de otro pueblo, como es relatado por un narrador que presencia la conversación de un grupo de hombres en un bar que: "empezaron a presumir que venían de cotorrearse a una vieja de La Matosa, una que había matado a su marido y que se las daba de muy bruja [...] que se le metieron a la casa y cómo la golpearon para que se estuviera quieta y pudieran cogérsela entre todos" (Melchor, 2017: 21). Como notoriamente ha argumentado la teórica argentina Rita Segato, la violación funciona como un acto alegórico del poder soberano que marca el cuerpo de la mujer para manifestar la conquista de un territorio. De acuerdo con esta interpretación, la violación de la Bruja vieja representa un "ritual sacrificial, violento y macabro" (Segato, 2013: 26) que constata la dominación y anexión de un 
territorio para el capitalismo transnacional. Como afirma Federici, comentando la teoría de Rita Laura Segato, el objetivo de la violencia contra las mujeres es:

to terrorize, to send a message, first to women and then, through them, to entire populations that no mercy should be expected. By clearing large territories of their inhabitants, by forcing people to leave their homes, their fields, their ancestral lands, violence against women is a crucial part of the operations of the mining and petroleum companies that today are displacing scores of villages in [...] Latin America (2018: 50).

Junto a este proceso de territorialización del capital, que ha tenido un impacto particularmente negativo en la vida campesina e indígena (Avila y Avila Romero, 2018: 140), estados ricos en petróleo como Veracruz han sido militarizados como parte de la guerra contra las drogas que ha aumentado el nivel de violencia y dado lugar a altos niveles de desplazamiento forzado. Zonas ricas en recursos naturales como Veracruz se han vuelto epicentro de la expansión de grupos criminales cuya presencia beneficia a corporaciones transnacionales que operan en un contexto en el cual empresas locales son desplazadas por la inseguridad causada por la acción delictiva de dichos grupos. Asimismo, la corrupción en las corporaciones policiacas produce una ambivalencia en la respuesta que oscila entre enfrentamiento y colaboración con los carteles que complica ulteriormente el escenario (Asoni, 2019: 16-19). Por estas razones, en los últimos ańos, Veracruz ha sido testigo de un dramático deterioro de las condiciones de vida de su gente y un aumento de las tasas de inseguridad y pobreza. Una combinación de poderes corporativos y criminales ha hecho de Veracruz un espacio emblemático de la depredación del capitalismo global, un espacio de precariedad donde los individuos y las comunidades 
vulnerables y desprotegidos se vuelven desechables. La novela referencia este escenario en las secciones finales cuando se cuenta del enfrentamiento entre el grupo liderado por el jefe de policía de La Matosa y los grupos criminales Raza Nueva y Grupo Sombra por el famoso tesoro que supuestamente la Bruja escondía en su casa:

Dicen que [...] lo que pasó fue que finalmente Rigorito y sus hombres sí encontraron el famoso tesoro escondido en la habitación de la Vieja [...]. Dicen que en algún momento [...] la codicia volvió loco a Rigorito y decidió matar a sus hombres antes de tener que compartir el botín con ellos. [...] Aunque también dicen que no [...] que era más seguro que los hombres de Rigorito [...] lo mataron a él primero; que lo que seguramente pasó fue que los policías se toparon con la avanzada de la Raza Nueva, que venía desde el norte barriendo la cochambre que dejó el Grupo Sombra en las estaciones petroleras, y que fueron ellos los que se chingaron a los policías y seguramente también al propio comandante, cuyo cadáver ya no tarda en aparecer en el sitio de alguna balacera, tal vez descuartizado también y con huellas de tortura y cartulinas con mensaje dirigidos al Cuco Barrabás y demás gente del Grupo Sombra (Melchor, 2017: 216).

El pasaje refleja la complejidad del escenario de guerra veracruzano con grupos criminales, paramilitares y fuerza del capital que se dividen el territorio progresivamente desplazando a las comunidades locales. Por lo tanto, el famoso tesoro de la Bruja aparece como representación metafórica del objeto de disputa: las tierras y el subsuelo veracruzano.

Las secciones de la novela dedicada a los acontecimientos relacionados a los personajes de Luismi y Norma, dos jóvenes de La Matosa, también ofrecen imágenes vívidas de la disgregación social que el sistema neoliberal ha producido en Veracruz. Estas 
secciones de la novela, elogiadas por el escritor boliviano Edmundo Paz Soldán como las más intensa y brillantes (2017), llaman la atención sobre los espacios de marginalidad donde fácilmente las personas pueden ser víctimas y victimarias al mismo tiempo.

Norma es una adolescente que abandona su casa para escapar de su padrastro Pepe, quien la violó en varias ocasiones. Después de fugarse, llega a La Matosa y, perdida y sola, conoce a Luismi, quien la acoge en su casa. Una vez que se descubre que Norma está embarazada, la llevan a la casa de la Bruja, donde la mujer realiza un aborto rudimentario ${ }^{1}$ que causa la muerte de la joven. Debido a que Norma aún no ha alcanzado la edad legal de consentimiento, Luismi es falsamente acusado de estupro contra a una persona menor de edad (a pesar de que el embarazo de Norma es el resultado de la violencia sexual cometida por Pepe) y perseguido por el corrupto cuerpo de policía de La Matosa. En fuga de la ley y del policía Rigorito, Luismi entra en contacto con el inframundo local y se involucra en hechos ilegales, como la organización del asalto a la casa de la Bruja (en la que también participa su padrastro, El Munra). Luismi representa la víctima no solo de un sistema judiciario corrupto sino también de un sistema socioeconómico que en espacios de precariedad tal como La Matosa, lo insta a perderse en el bajo mundo criminal, ya que las únicas oportunidades son trabajar por un poderoso "Partido" político que paga "doscientos varos por días [...] más comestibles a granel que entregaban cada semana” (Melchor, 2017: 73) o la prostitución. Es en este último contexto que conoce a un ingeniero que le promete una "dichosa chamba de la Compañía [...] en los campos petroleros de Palogacho, una chamba de técnico [...] con todas las prestaciones" (Melchor, 2017: 75). Sin embargo, "aquello no pasaría nunca, porque hacía años ya que la Compañía no contrataba a nadie que no fuera

${ }^{1}$ Cabe señalar que en el estado de Veracruz el aborto está prohibido. 
pariente directo o recomendado de los líderes sindicales" (Melchor, 2017: 75).

En los párrafos mencionados, la novela aborda el tema la dependencia de algunas áreas del estado de Veracruz de la industria extractiva y de la falta de inversión social para la población local depauperada por el modelo neoliberal. Es en este contexto social que Luismi pierde el control de su vida y cuando el jefe de policía Rigorito y sus hombres detienen y torturan a Brando, uno de los hombres que habían participado en el asalto y asesinato de la Bruja, este último afirma que "el que la mató fue Luismi; la culpa de todo fue de Luismi; él fue quien le enterró el cuchillo en el cuello" (Melchor, 2017: 158). Sin embargo, el tesoro de la Bruja no es el móvil del asesinato. Lo que mueve a Luismi es la necesidad de validar su virilidad frente a la pandilla, ya que sus amigos lo acosan por tener una relación sexual con un ingeniero de la Compañía petrolera que opera en el área. Lo que desencadena la furia de Luismi no es, como Brando cree y le cuenta a Rigorito, el hecho de que Bruja podría tener dinero escondido, sino que "la Bruja se había dado cuenta de que el Luismi estaba bien clavado con ese ruco, que no paraba nunca de hablar de él y de lo chingón que era y de la chamba que según iba a conseguirle en la Compañía Petrolera" (Melchor, 2017: 187). Si bien Luismi es realmente responsable del horrendo e insensible crimen, por otro lado, su crimen también es el resultado de "la creciente militarización de la vida cotidiana, con su consiguiente glorificación de modelos agresivos y misóginos de masculinidad" (Federici, 2018: 56) que se ha impuesto con la creciente militarización de la región:

the proliferation of armed men and the development of a new sexual division of labor, whereby most jobs open to men (as private domestic guards, commercial security guards, prison guards, members of gangs and mafias, and soldiers in regular or private 
armies) require violence, plays a central role in forging increasingly toxic masculinities (Federici, 2018: 56).

El crimen cometido por Luismi es, entonces, "una cuestión de afirmar una dominación existente" (Segato, 2010: 79), un acto ritual de validación de su control viril subyugando un cuerpo femenino. A través de los cuerpos de las Brujas y de Norma, subyugados por poderes oscuros, la novela sitúa el tema del cuerpo femenino explotado en el contexto del afianzamiento del capitalismo global neoliberal en un espacio emblemático de depredación del capitalismo post-TLCAN en México. Es por esta razón que la muerte de la Bruja, así como la verdad sobre la muerte de Norma, son casos que no solo nadie realmente busca resolver, sino que, a la autoridad, representada en la novela por el comandante Rigorito, "le valía tres toneladas de verga, y lo único que el culero quería saber era dónde estaba el oro" (Melchor, 2017: 157), palabras que evidencian el descuido social arraigado al sistema neoliberal. Como Mesha Maren escribe en su reseña de la novela, comparándola con Blood Meridian de Cormac McCarthy, Temporada de huracanes "illuminates the current state of post-NAFTA globalization that leaves ordinary small-town communities just as vulnerable to modern day scalp-hunters and bloodthirsty capitalists in all their various forms" (2020). Lo que la novela evidencia, por lo tanto, son las formas en las cuales el capitalismo global, los cazadores de escalpo o de tesoro, en este caso, arrasan las pequeñas comunidades de la provincia mexicana. 


\section{Bibliografía}

Asoni, Ettore, 2019, "Drug Wars and the Neoliberalization of the Space in Latin America", Diacronie. Studi di Storia Contemporanea: Mafia e storiografia. Premesse culturali e prospettive attuali, núm. 39, pp. 1-25.

Avila Agustín y Avila Romero, León Enrique, 2017, "Las nuevas Zonas Económicas Especiales en México: despojo agrario y resistencia campesina”, Revista Nera, vol. 20, núm. 40. pp. 138162.

Badillo, Alejandro, 2014, "La violencia de los sentidos", Crítica. Disponible en: http://revistacritica.com/contenidos-impresos/ vigilia/falsa-liebre-de-fernanda-melchor-por-alejandro-badillo.

Checa-Artasu, Martin, 2014, "Geografía, poder y petróleo en México. Algunos ejemplos", Scripta Nova: Revista Electrónoca de Geografía y Ciencias Sociales, año xvıII, vol. 51, núm. 495, pp. $1-15$.

Deckard, Sharae. 2016, "The Political Ecology of Storms in Caribbean Literature", en Chris Campbell y Michael Niblett (comps.), The Caribbean Aesthetics, World-Ecology, Politics, Liverpool University Press, Liverpool, pp. 25-45.

Federici, Silvia, 2004, Calibán y la bruja: mujeres, cuerpo y acumulación originaria, Traficantes de sueños, Madrid.

land.

, 2018, Witches, Witch-Hunting and Women, pm Press, Oak-

García Márquez, Gabriel, 1967, Cien años de soledad, Alfaguara, Ciudad de México. [Versión epub].

Ghosh, Amitav, 1992, "Petrofiction: The Oil Encounter and the Novel", The New Republic, pp. 29-33. 
Harvey, David, 2004, “The 'New’ Imperialism: Accumulation by Dispossession”, The Socialist Register, núm. 40.

Herlinghaus, Hermann, 2013, Narcoepics: A Global Aesthetics of Sobriety, Bloomsbury, Londres.

Jameson, Frédric, 1981, The Political Unconscious: Narrative as a Social Symbolic Act, Methuen, Londres.

Maren, Mesha, 2020, “'Hurricane Season’ Is Like '2666’ Meets Hieronymus Bosch", Southern Review of Books. Disponible en: https://southernreviewofbooks.com/2020/04/15/hurrricane-season-fernanda-melchor-review/.

Melchor, Fernanda, 2008, Mi Veracruz, Ayuntamiento de Veracruz, Veracruz.

2013, Aqui no es Miami, Literatura Random House, Ciudad de México.

, 2013, Falsa liebre, Almandía, Ciudad de México.

, 2017, Temporada de huracanes, Literatura Random House, Ciudad de México.

Nixon, Robert, 2011, Slow Violence and the Environmentalism of the Poor, Harvard University Press, Cambridge.

Ortuño, Antonio, 2017, "Por fin”, Letras Libres. Disponible en: http:/www.letraslibres.com/espana-mexico/revista/por-fin.

Paley, Dawn, 2014, Drug War Capitalism, AK Press, Oakland.

Paz Soldán, Edmundo, 2017, "Fernanda Melchor: intensidad y horror", Latercera. Disponible en: http://www.latercera.com/ voces/fernanda-melchor-intensidad-horror/.

Prado, Luis, 2013, "Las raíces negras de Veracruz", El País. Disponible en: https://elpais.com/elpais/2013/07/25/ eps/1374766765_208709.html.

Riddle, Amy, 2018, "Petrofiction and Political Economy in the Age of Late Fossil Capital", Mediations, vol.2, núm. 31, pp. 
55-74. Disponible en: www.mediationsjournal.org/articles/ petrofiction.

Sánchez-Prado, Ignacio, 2020, “Fernanda Melchor's Hurricane Season: A Literary Triumph on the Failures of Mexican Modernization", Words Without Borders. Disponible en: https://www.wordswithoutborders.org/book-review/fernanda-melchors-hurricane-season-a-literary-triumph-ignacio-m-sanchez-pra.

Segato, Rita Laura, 2010, "Territory, Sovereignty, and Crimes of the Second State", en Terrorizing Women: Feminicide in the Américas, Rosa-Linda Fregoso y Cynthia Bejarano (coords.), Duke University Press, Durham, pp. 70-92.

, 2013, La escritura en el cuerpo de las mujeres asesinadas en Ciudad Juárez, Tinta Limón, Buenos Aires.

Szeman, Imre, 2012, "Petrofictions", American Book Review, p. 3.

Tuan, Yi-Fu, 2013, Landscape of Fear, Minnesota University Press, Minneapolis.

Wolfson, Gabriel, 2017, “Temporada de huracanes de Fernanda Melchor”, Critica. Disponible en: http://revistacritica.com/ contenidos-impresos/vigilia/temporada-de-huracanes-de-fernanda melchor-gabriel-wolfson.

Zavala, Oswaldo, 2014, "Imagining the U.S.-Mexico Drug War: The Critical Limits of Narconarratives", Comparative Literature, vol. 3, núm. 66, pp. 340-360.

, 2016, "Crónicas despolitizadas: Seguridad, política y los imaginarios periodísticos sobre el narco en México", en En camas separadas: Historia y literatura en el México del siglo XXI, David Miklos (coord.), Tusquets, Ciudad de México, pp. 193224. 